Syvolap Victor, Zemlianiy Yaroslav, Brick Andrey, Bryleva Vera. Relationship between levels of Copeptin, NTproBNP and structural functional parameters of the heart in patients with acute myocardial infarction with ST-segment elevation complicated by pulmonary hypertension. Journal of Education, Health and Sport. 2020;10(2):126-132. eISSN 2391-8306. DOI http://dx.doi.org/10.12775/JEHS.2020.10.02.017 https://apcz.umk.pl/czasopisma/index.php/JEHS/article/view/JEHS.2020.10.02.017 https://zenodo.org/record/3678376

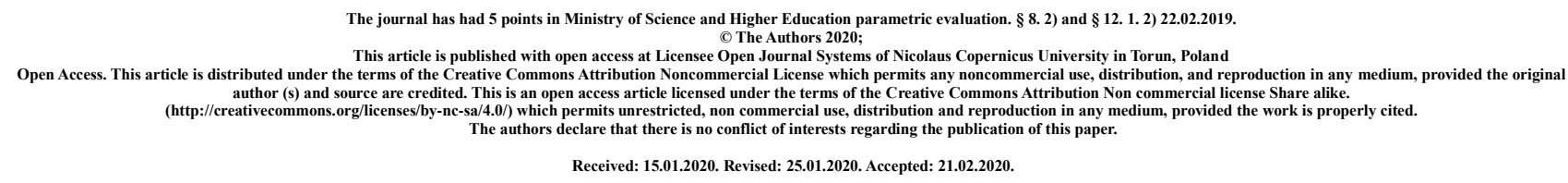

\title{
Relationship between levels of Copeptin, NTproBNP and structural functional parameters of the heart in patients with acute myocardial infarction with ST-segment elevation complicated by pulmonary hypertension
}

\author{
${ }^{1}$ Victor D. Syvolap, ${ }^{1}$ Yaroslav V. Zemlianiy, ${ }^{2}$ Andrey V. Brick, ${ }^{2}$ Vera P. Bryleva \\ 1 «Zaporozhye State Medical University» (Ukraine) \\ 2 «City hospital and emergency ambulance" Zaporizhia city council (Ukraine)
}

\begin{abstract}
Introduction: Acute Myocardial Infarction with ST-Segment Elevation (STEMI) - one of the major causes of morbidity and mortality worldwide, including in Ukraine. The prevalence of pulmonary hypertension (PH) in STEMI significantly is not known due to lack of epidemiological studies in this area. Currently, the prognostic significance of PH in patients with STEMI is not set.

Objective: To evaluate the levels of NTproBNP and Copeptin and their relationship with structural and functional features of the heart in patients with STEMI complicated by $\mathrm{PH}$.

Materials and Methods: The study involved 85 patients (49 men and 36 women) with STEMI who were treated in intensive care and emergency cardiology for the treatment of patients with myocardial infarction communal non-profit enterprise "City hospital and emergency ambulance" Zaporizhia city council. Patients were divided into two groups: 53 patients with STEMI in the presence of PH (mean age 72,40 \pm 2,26 years), 32 patients with STEMI without PH (mean age 67,30 $\pm 2,12$ years).

Results: In assessing the clinical anamnesis (Table 1) found that PH mainly develops in the anterior localization of STEMI (73,6\%). In patients with STEMI in the presence of PH compared with patients without significant PH frequently observed violations systolic function $(64,2 \%)$. Significant increasing in the level of copeptin $(\mathrm{p}<0,05)$ were found in the serum of patients with acute myocardial infarction in the presence of PH compared with patients with normal pressure in the pulmonary artery (PA). NTproBNP level was also higher in patients with STEMI and PH compared with patients without PH.
\end{abstract}


In patients with STEMI and PH we found reliable positive correlation between the NTproBNP and copeptin level $(+0,31 ; \mathrm{p}<0,05)$, systolic pressure in PA $(0,41 ; \mathrm{p}<0,05)$ and negative correlation between NTproBNP level and ejection fraction $(-0,41 ; \mathrm{p}<0,05)$, stroke volume $(-0,42 ; \mathrm{p}<0,05)$. Copeptin positively correlated with the level of NTproBNP $(+0,31 ; p<0,05)$, systolic pressure in PA $(0,37 ; \mathrm{p}<0,05)$ and negatively correlated with ejection fraction $(-0,30 ; \mathrm{p}<0,05)$.

Conclusions: Pulmonary hypertension develops mainly in the anterior localization of STEMI (73,6\%) in the presence of systolic dysfunction (64,2\%). In patients with STEMI and PH level of NTproBNP and copeptin in serum were increased compared than patients without PH. In patients with STEMI and PH NTproBNP and copeptin levels correlated with decreased left ventricular ejection fraction and increased pressure in the pulmonary artery.

Key words: acute myocardial infarction, pulmonary hypertension, copeptin, NTproBNP

\section{INTRODUCTION}

Acute Myocardial Infarction with ST-Segment Elevation (STEMI) - one of the major causes of morbidity and mortality worldwide, including in Ukraine [1]. The prevalence of pulmonary hypertension (PH) in STEMI significantly is not known due to lack of epidemiological studies in this area [2]. Currently, the prognostic significance of PH in patients with STEMI is not set.

In recent studies it has been shown that mortality in non-STEMI was not significantly dependent on the availability of PH [3]. However, in patients with increased pulmonary artery pressure more reliably detected cardiogenic shock and respiratory failure.

In patients with STEMI pulmonary hypertension develops mainly due to congestive heart failure [4]. Therefore, evaluating of NTproBNP clinical value, the known marker of heart failure (HF), in patients with STEMI in the presence of PH is actual. It is known, that the level of this marker is an independent risk factor for negative events in $\mathrm{PH}$ [2].

In recent years researches focuses on the study of diagnostic and prognostic value copeptin in patients with acute myocardial infarction [5]. It is proved, that patients with STEMI has activation of vasopressin, a fragment of which is copeptin. Researchers found it predictive properties for the development of heart failure and sudden cardiac death in these patients $[6,7,8]$. The role of the association copeptin, NTproBNP and heart remodelling in patients with myocardial infarction complicated by increased pressure in the pulmonary artery has not been investigated.

It is important to determine the role of copeptin and NTproBNP and their relationship with the structural and functional parameters of the heart in patients with STEMI in the presence of PH.

\section{OBJECTIVE}

To evaluate the levels of NTproBNP and Copeptin and their relationship with structural and functional features of the heart in patients with STEMI complicated by PH.

\section{MATERIALS AND METHODS}

The study involved 85 patients (49 men and 36 women) with STEMI who were treated in intensive care and emergency cardiology for the treatment of patients with myocardial infarction communal non-profit enterprise "City hospital and emergency ambulance" Zaporizhia city council. Patients were divided into two groups: 53 patients with STEMI in the presence of PH (mean age 72,40 \pm 2,26 years), 32 patients with STEMI without $\mathrm{PH}$ (mean age 67,30 $\pm 2,12$ years). 
The diagnosis of myocardial infarction raised in case of typical clinical manifestations, increased troponin I level in blood, typical ECG and echocardiography changes according to the protocol number 455 of 07/02/2014 Ministry of Health of Ukraine.

To determine the level of troponin we used semi-automated photoelectric fotomer FAX 303 Stat Plus (ZOMZ, Russia) with a set of Hema (troponin I-ELISA, HEMAtest, Germany).

Inclusion criteria: patients with STEMI with or without PH.

Exclusion criteria: presence of cancer, pulmonary arterial due to diseases of the respiratory system.

All patients were examined during the first three days using two-dimensional echocardiography in the machine "MyLab50" ( "Esaote", Italy) by American Society of Echocardiography recommendation [9]. The study determined the following parameters: size of the left atrium (LA), the size of the left ventricle in systole (LVDs) and diastole (LVDd), diastolic thickness of the interventricular septum (IVSd) and back wall (PWd), the size of the right ventricle (RV) and right atrium (RA). Based on the data we calculated left ventricular ejection fraction (EF) and LV stroke volume (SV), myocardial mass (MM) of LV and myocardial mass index (MMI) LV and relative wall thickness (RWT). Systolic pulmonary artery pressure (systPAP) determined by recommendations for diagnosis and treatment of pulmonary hypertension European Society of Cardiology and the European Respiratory Society 2015 [2]. Pulmonary hypertension was diagnosed in identifying systolic pulmonary artery pressure above 30 mmHg.

Mode pulse Doppler parameters were determined following transmitral flow: the maximum speed of early diastolic filling (MVE) and maximum speed filling the left ventricle during systole of the left atrium (MVA), their ratio (MV E / A) and isovolumetric relaxation time (LV IVRT).

Diastolic left ventricular function was determined by the recommendations of the American Society of Echocardiography [10].

Statistical analysis of the data was performed using the statistical software package "Statistica 6.0 for Windows" (StatSoft Inc., № AXXR712D833214FAN5). To determine the normal distribution using criteria Shapiro-Wilkie. Given the type of distribution (normal or not) variables are presented as $\mathrm{M} \pm$ $\mathrm{m}$ or Me $(25-75 \%)$. To determine the reliability of the differences between the parameters used double t-test for independent samples by Student (with a normal distribution) and Wilcoxon criterion with uneven distribution. Evaluation of relationships between parameters was performed using the method of correlation analysis by Pearson and Spearman. The reliability of the differences between the figures stated at $\mathrm{p}<0.05$. All tests were two sided.

\section{RESEARCH RESULTS}

In assessing the clinical anamnesis (Table 1) found that PH mainly develops in the anterior localization of STEMI $(73,6 \%)$. In patients with STEMI in the presence of PH compared with patients without significant $\mathrm{PH}$ frequently observed violations systolic function $(64,2 \%)$.

In $20,8 \%$ of patients with STEMI and PH ventricular aneurysm was found, which was significantly different from group without PH. Significant differences between groups on the prevalence of arterial hypertension and diabetes mellitus were not found. Gender and age differences between patients with STEMI depending on the availability of PH also not determined. 
Clinical characteristics of patients with STEMI depending on the availability of PH

\begin{tabular}{|c|c|c|}
\hline The indicator units & STEMI with PH $(\mathrm{n}=53)$ & STEMI without $\mathrm{PH}(\mathrm{n}=32)$ \\
\hline Age, years & $72,40 \pm 2,26$ & $67,30 \pm 2,12$ \\
\hline Men, n (\%) & $30(56.6 \%)$ & $19(59.4 \%)$ \\
\hline Women, n (\%) & $23(44.4 \%)$ & $13(40.6 \%)$ \\
\hline Infarction posterior localization, $\mathrm{n}(\%)$ & $14(26.4 \%)$ & $15(46.9 \%)$ \\
\hline Infarction anterior localization, n (\%) & $39(73.6 \%) *$ & $17(53.1 \%)$ \\
\hline Systolic dysfunction, $\mathrm{n}(\%)$ & $34(64.2 \%) *$ & $13(40.6 \%)$ \\
\hline LV aneurysm, n (\%) & $11(20.8 \%) *$ & $3(9.4 \%)$ \\
\hline Diabetes mellitus type $2, \mathrm{n}(\%)$ & $14(26.4 \%)$ & $6(18.8 \%)$ \\
\hline Arterial hypertension, n (\%) & $46(86.8 \%)$ & $27(84.3 \%)$ \\
\hline HF functional class I, II, n (\%) & $14(25 \%)$ & $18(56.3 \%)$ \\
\hline HF functional class III, IV, n (\%) & $42(75 \%) *$ & $14(43.7 \%)$ \\
\hline Left brunch blockade, $\mathrm{n}(\%)$ & $6(11.3 \%)$ & $2(6.25 \%)$ \\
\hline
\end{tabular}

Note: * - significant differences with group STEMI without $\mathrm{PH}(\mathrm{p}<0,05)$.

Significant increasing of the copeptin level $(\mathrm{p}<0,05)$ were found in the serum of patients with acute myocardial infarction in the presence of $\mathrm{PH}$ compared with patients with normal pressure in the pulmonary artery. NTproBNP level was also higher in patients with STEMI and PH compared with patients without $\mathrm{PH}$ (Table. 2).

Table 2

Levels of Copeptin and NTproBNP in patients with STEMI depending on the availability of PH

\begin{tabular}{|c|c|c|}
\hline The indicator units & STEMI with PH $(\mathrm{n}=53)$ & STEMI without PH $(\mathrm{n}=32)$ \\
\hline Copeptin $\mathrm{mg} / \mathrm{ml}$ & $4,58(1.74 ; 4.32)^{*}$ & $2,61(1.55 ; 3.17)$ \\
\hline NTproBNP,pg / ml & $140,38(43.41,174.78)^{*}$ & $73,91(11.44 ; 90.52)$ \\
\hline
\end{tabular}

Note: * - significant differences with group STEMI without PH $(\mathrm{p}<0,05)$.

In patients with STEMI revealed the presence of PH we found reliable direct correlation (Table. 3) between the level of NTproBNP and copeptin $(+0,31 ; p<0,05)$, systolic pressure in PA $(0.41 ; p<0,05)$ and negative correlation between the level of NTproBNP and EF $(-0,41 ; p<0,05), S V(-0,42 ; p<0,05)$. Copeptin positively correlated with the level of NTproBNP $(+0,31 ; p<0,05)$, systolic pressure in PA $(0,37 ; \mathrm{p}<0,05)$ and negatively correlated with ejection fraction $(-0,30 ; \mathrm{p}<0,05)$. 


\section{Table 3}

Correlation levels of Copeptin, NTproBNP, clinical signs and structural-functional parameters of the heart

\begin{tabular}{|l|c|c|}
\hline \multirow{2}{*}{\multicolumn{1}{|c|}{ Indicator }} & Copeptin pg / ml & NTproBNP, pg / ml \\
\cline { 2 - 3 } & $\mathrm{r}$ & $\mathrm{r}$ \\
\hline Copeptin pg / ml & - & $-31^{*}$ \\
\hline NTproBNP, pg / ml & $0,31 *$ & $-0,41 *$ \\
\hline EF \% & $-0,30 *$ & $-0,42 *$ \\
\hline EO, ml & 0,25 & 0,18 \\
\hline LVDd, sm & 0,12 & 0,21 \\
\hline RVd, sm & 0,04 & $0,41 *$ \\
\hline Systolic PA pressure, $\mathrm{mmHg}$ & $0,37 *$ & \\
\hline
\end{tabular}

Notes: significant correlation $-*(\mathrm{p}<0,05)$.

\section{DISCUSSION OF RESEARCH RESULTS}

According to our study in patients with STEMI with increased pressure in the PA infarction usually develops in anterior wall and interventricular septum. This is due to the influence of systolic dysfunction and the formation of heart failure, which is typical for anterior localization of myocardial infarction. In recent studies researches showed that $\mathrm{PH}$ in diseases of the left chambers of the heart develops usually in the presence of congestive heart failure [11].

In patients with STEMI and PH we found increased levels of NTproBNP and copeptin in serum. According Nickel N. level of Copeptin also increases in patients with PH compared with patients with normal pressure in the pulmonary artery [13]. The authors found a correlation with the level of copeptin and NTproBNP, functional class of heart failure and glomerular filtration rate. Level of Copeptin became independent predictor of adverse prognosis in patients with $\mathrm{PH}$ [12]. According to our study in patients with STEMI and PH Copeptin levels associated with decreased left of ventricular systolic function, heart failure functional class and increased pressure in the PA. Consequently, the using of Copeptin and NTproBNP may be perspective for risk stratification and assessment of severity of patients with STEMI in the presence of PH.

\section{CONCLUSIONS}

1. Pulmonary hypertension develops mainly in the anterior localization of STEMI $(73,6 \%)$ in the presence of systolic dysfunction $(64,2 \%)$.

2. In patients with STEMI and PH levels of NTproBNP and copeptin in serum increased compared with patients without PH.

3. In patients with STEMI and PH levels of NTproBNP and copeptin in serum correlated with decreased left ventricular ejection fraction and increased pressure in the pulmonary artery.

Prospects for further research. The study revealed changes copeptin and NTproBNP levels and their relationship with structural and functional parameters of the heart in patients with acute myocardial infarction in the presence of $\mathrm{PH}$. In the future, it is advisable to continue to study the prognostic significance of copeptin and NTproBNP levels in patients with STEMI and PH.

Conflict of interest. Conflict of interest is absent. 


\section{LITERATURE}

1. V.M.Kovalenka Sci. E. Lutay, Yu.M.Sirenko. Cardiovascular disease. The classification standards for diagnosis and treatment. K .: Association of Cardiologists of Ukraine. 2016; 128 p. Ukrainian.

2. Galiè N., Humbert M., Vachiery JL 2015 ESC / ERS Guidelines for the diagnosis and treatment of pulmonary hypertension: The Joint Task Force for the Diagnosis and Treatment of Pulmonary Hypertension of the European Society of Cardiology (ESC) and the European Respiratory Society (ERS): Endorsed by: Association for European Paediatric and Congenital Cardiology (AEPC), International Society for Heart and Lung Transplantation (ISHLT). Eur. Heart J. 2016; 37: 67-119. doi: 10.1093 / eurheartj / ehv317.

3. Ahsan S., Hamed S. The impact of pulmonary hypertension on in-hospital outcomes of non-st elevation myocardial infarction. J. Am. Coll. Cardio. in 2018; 71: 1940. doi: 10.1016 / S0735-1097 (18) 32481-1.

4. Guazzi M., Borlaug BA Pulmonary Hypertension Due to Left Heart Disease. Circulation. 2012; 125: 975-990. doi: 10.1161 / CIRCULATIONAHA.111.085761.

5. Boeddinghaus J., Reichlin T., Nestelberger T. Early diagnosis of acute myocardial infarction in patients with mild elevations of cardiac troponin. Clin Res Cardiol. 2017; 106 (6): 457- 467. doi: 10.1007 / s00392-016-1075-9.

6. Smaradottir MI, Ritsinger V., Gyberg V. Copeptin in patients with acute myocardial infarction and newly detected glucose abnormalities - A marker of increased stress susceptibility? A report from the Glucose in Acute Myocardial Infarction cohort. DiabVasc Dis Res. in 2017; 14 (2): 69- 76. doi: 10.1177 / 1479164116664490. PMID: 28118730.

7. Silva Marques J., Marques, Luz-Rodrigues H., David C. Biomarkers of functional class in systolic heart failure: the relevance of copeptin. Rev Port Cardiol. 2012; (31): 701-1105. doi: 10.1016/j.repc.2012.09.001.

8. Vetrone F., Santarelli S., Russo V. Copeptin decrease from admission to discharge has favorable prognostic value for the 90-day events in patients admitted with dyspnea. Clin Chem Lab Med. 2014; 52 (10): 1457-64. doi: 10.1515/cclm-2014-0207.

9. Lang RM, Badano LP, Mor-Avi V. Recommendations for cardiac chamber quantification by echocardiography in adults: an update from the American Society of Echocardiography and the European Association of Cardiovascular Imaging. J. Am. Soc. Echocardiogr. 2015; 28: 1-39. doi: 10.1016 / j.echo.2014.10.003.

10. Nagueh SF, Smiseth OA, Appleton CP Recommendations for the Evaluation of Left Ventricular Diastolic Function by Echocardiography: An Update from the American Society of Echocardiography and the European Association of Cardiovascular Imaging. J. Am. Soc. Echocardiogr. in 2016; 29: 277-314. doi: 10.1016 / j.echo.2016.01.011.

11. Mehra P., Mehta V., Sukhija R. Pulmonary hypertension in left heart disease. Arch. Med. Sci. in 2019; 15: 262-273. doi: 10.5114 / aoms.2017.68938.

12. Nickel NP, Lichtinghagen R., Golpon H. Circulating levels of copeptin predict outcome in patients with pulmonary arterial hypertension. Respir Res. in 2013; 14: 130. doi: 10.1186 / 1465-992114-130. 

About the Authors:
Syvolap Victor Denisovich, MD, Professor, Head of the Department of Internal Diseases 1. ZSMU. Tel. +380501363068. Almost Email: svd.zgmu@ gmail.com. ORCID 0000-0002-7342-9065
Zemlianiy Yaroslav Vadimovich, PD, assistant of the Department of Internal Medicine 1. ZSMU. Tel . +380 678785 835. Almost Email: jarlord@ gmail.com. ORCID 0000-0002-6494-6570
Brick Andrey Vasilievich, head of the Department of Cardiology, «City hospital and emergency ambulance" Zaporizhia city council. Tel . +380 975552152. Almost Email: brickav@ gmail.com.
Bryleva Vera Petrovna, doctor of the Department of Cardiology, «City hospital and emergency ambulance" Zaporizhia city council. Tel .+380 684511095. Almost Email: brylevavp@ gmail.com.
Address for correspondence
Zemlianyi Yaroslav Vadimovich
Department of Internal Medicine 1 ZSMU
Tel . 380678785835 .
e-mail:jarlord@gmail.com. 